\title{
DORMANCIA EN SEMILLAS DE PAPAYA CV MARADOL ROJA DURANTE EL ALMACENAMIENTO ${ }^{1}$
}

\author{
Maruchi Alonso-Esquivel ${ }^{2}$, Yoleinis Ortiz-López $z^{3}$, Roberto Ramos-Ramírez ${ }^{3}$,Hugo Oliva-Diaz ${ }^{2}$, \\ Maricela Capote-del Sol ${ }^{2}$
}

\section{RESUMEN}

Dormancia en semillas de papaya cv Maradol Roja durante el almacenamiento. El objetivo del presente trabajo fue determinar el efecto de la humedad de la semilla cultivar Maradol Roja y la temperatura durante el almacenamiento, en la dormancia de la papaya. Este trabajo fue desarrollado en el Laboratorio Nacional de Semillas de la Empresa Productora y Comercializadora de Semillas, provincia La Habana, Cuba, en el período noviembre 2006 a enero 2008. Fueron extraídas las semillas de frutos de papaya cv Maradol Roja, y se secaron a tres contenidos de humedad (12\%, $10,56 \%$ y $9,26 \%$ ), y acondicionadas en bolsas de polietileno y almacenadas a $15^{\circ} \mathrm{C}$ y $4^{\circ} \mathrm{C}$. El porcentaje de germinación, altura y peso fresco de la planta fueron realizadas al inicio y después de tres, seis, nueve y doce meses de almacenamiento. Hubo tendencia a la disminución de la germinación en los primeros seis meses del almacenamiento, alcanzándose los valores más bajos del porcentaje de germinación $(55,6 \%)$. A partir de los nueve y doce meses fue detectado un aumento significativo en la germinación, independiente del contenido de humedad de la semilla. El ambiente de $15^{\circ} \mathrm{C}$ favoreció mayor germinación en períodos mayores de almacenamiento en relación a las mantenidas a $4^{\circ} \mathrm{C}$, que exhibieron mejores porcentajes de germinación en un período menor (tres meses de almacenamiento). La dormancia finalizó en los periodos de almacenamiento a los nueve y doce meses, independientemente del contenido de humedad.

Palabras claves: Carica papaya L., germinación, sarcostesta.

Key words: Carica papaya L., germination, sarcostesta.

\section{ABSTRACT}

Dormancy of papaya seeds cv Maradol Roja during storage. The objective of this work was to determine the effect of humidity content of papaya cv Maradol Roja and storage temperatura on dormany. The work was conducted at the Laboratorio Nacional de Semillas, Empresa Productora y Comercializadora de Semillas, La Habana, Cuba, from November 2006 to January 2008. Seeds were extracted from papaya fruits cv Maradol Roja and dried to three humidiy contents $(12 \%, 10,56 \%$ y $9,26 \%)$, and aconditioned in polyiethylene bags and stored at $15^{\circ} \mathrm{C}$ and $4^{\circ} \mathrm{C}$. Percentaje of seed germination, heigth and fresh weight of the seedlings were measured at 3, 6,9 and 12 months in storage. There was a trend towards reduced seed germination during the first 6 months in storage $(55,6 \%)$. After 9 and 12 months a significant incresase in germination was observed, independent of seed water content. Seeds stored in environment at $15^{\circ} \mathrm{C}$ favored germination after longer sotrage compared to seeds kept in storage for shorter periods (3 months). Seed dormany concluded between 9 and 12 months in storage irrespective of seed water content.
\end{abstract}

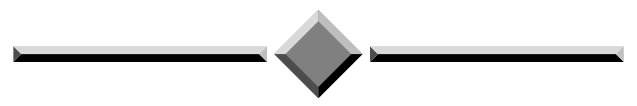

Recibido: 13 de junio, 2009. Aceptado: 3 de octubre, 2011.

2 Instituto de Investigaciones en Fruticultura Tropical. Ave. 7ma. No. 3005 entre 30 y 32, Miramar, Playa. Ciudad de La Habana. Cuba, C.P. 11300.Telefax: (53-7) 2046794, Teléfono: (53-7) 209358.mejoramiento@iift.cu; certificacion@iift.cu; onp_palma@iift.cu

3 Laboratorio Nacional de Semillas. Empresa Productora y Comercializadora de Semillas. MINAG. Ave Conill, esq ave de Independencia. Cuba.esdir@enet.cu 


\section{INTRODUCCIÓN}

La papaya (Carica papaya L.) es un frutal que se propaga por semillas, siendo un proceso práctico, económico y muy utilizado por los agricultores. La calidad fisiológica de las semillas de papaya está caracterizada por la germinación y el vigor, considerando que son factores de gran importancia a nivel de plantación (Lopes y Souza 2008).

La conservación de semillas ofrece un soporte a los trabajos de mejoramiento, posibilita el intercambio de germoplasma y principalmente, la preservación de la viabilidad genética (Aroucha et al. 2007).

Dentro de los factores que afectan el mantenimiento de la calidad de la semilla a lo largo de un periodo determinado, se destaca el grado de humedad, la condición del ambiente de almacenamiento (principalmente, la temperatura y la humedad relativa del aire) así como el tipo de embalaje utilizado (Martins et al. 2004). La longevidad de las semillas está influenciada por otros factores tales como la especie, variedad, el historial del lote de semillas y estado de madurez del fruto (Carvalho y Nakagawa 2000).

Las semillas en general necesitan niveles adecuados de oxígeno, humedad y temperatura para germinar. Para lograr una adecuada plantación se requiere contar con semillas de buena calidad, y se hace necesario conocer su capacidad de germinación (Berbert et al. 2008).

Las informaciones relacionadas con la conservación de semillas de papaya no se encuentran bien definidas, y en ocasiones son contradictorias debido principalmente a que no se precisan las condiciones de almacenamiento. La mayoría de los trabajos indican que las semillas de papaya pierden su viabilidad en periodos cortos de almacenamiento (Yahiro 1979, Santos et al. 1999). Pero Bass (1975) observó que las semillas de papaya mantuvieron su viabilidad de manera satisfactoria hasta los seis años de almacenamiento.

El presente trabajo tiene como objetivo determinar el efecto de la humedad de la semilla cv Maradol Roja y la temperatura durante el almacenamiento, en la dormancia de la papaya.

\section{MATERIALES Y MÉTODOS}

Este trabajo fue desarrollado en el Laboratorio Nacional de Semillas de la Empresa Productora y Comercializadora de Semillas, provincia la Habana, Cuba en el periodo noviembre 2006 a enero 2008. Las semillas fueron obtenidas de frutos hermafroditas del cultivar comercial "Maradol Roja", cosechados con grado de maduración III ( $3 / 4$ de la superficie de la fruta de color amarillo) a partir de una plantación ubicada en un campo experimental de dicha empresa.

Después de la recepción de los frutos, el lote fue dividido en tres sub-lotes, que fueron manejados separadamente. Las semillas fueron retiradas de los frutos y lavadas con agua limpia para la eliminación de la placenta o residuos de pulpa. Posteriormente, se procedió a la eliminación manual de la sarcotesta siguiendo la metodología de Ramos y Ramos (2002). Finalmente, las semillas fueron escurridas y secadas a la sombra sobre hojas dobles de papel de filtro en un local ventilado.

El proceso de secado fue realizado hasta que las semillas alcanzaran el contenido de humedad establecido para cada sub-lote. De esta forma, se trabajó con los contenidos de humedad de: $12 \%, 10,56 \%$ y $9,26 \%$ según las recomendaciones de Viggiano et al. (2000). Para alcanzarlos se realizó el monitoreo del contenido de humedad de la semilla, que consistía en: muestreo de las semillas después de un periodo de secado, acondicionamiento de las semillas restantes en recipientes impermeables, determinación del contenido de humedad de las semillas muestreadas y continuación del secado de las restantes.

Una vez alcanzado el contenido de humedad deseado, las semillas fueron acondicionadas en bolsas de polietileno recubiertas con películas de aluminio y conservadas en dos condiciones ambientales: cámara fría con temperatura de $15^{\circ} \mathrm{C}$ (A1) y refrigerador con temperatura de $4^{\circ} \mathrm{C}$ (A2) durante cuatro periodos de almacenamiento (PA3: tres meses, PA6: seis meses, PA9: nueve meses y PA12: doce meses), a partir de enero del 2007. En cada ambiente se utilizó un termómetro para el control de la temperatura, donde fueron realizadas tres lecturas diarias. 
La calidad fisiológica de las semillas fue evaluada mediante la prueba de germinación, peso fresco (gramos) y altura de la planta (centímetros) y fueron realizadas inmediatamente después del secado de las semillas y durante los cuatro periodos de almacenamiento.

La prueba de germinación se realizó según las reglas para el análisis de semillas siguiendo la metodología establecida por la Empresa de Semillas Varias (MINAG 1996) y se utilizaron cuatro muestras de 50 semillas por repetición para cada sub-lote. Estas semillas fueron distribuidas en rollos de papel "Germitest", humedecidos con agua destilada equivalente a 2,5 veces el peso del sustrato seco (Ramos y Ramos 2002). Los rollos de papel se colocaron en cámaras de germinación tipo B.O.D, regulada a temperaturas alternas de $20-30^{\circ} \mathrm{C}$ (8-16 horas).

El diseño experimental utilizado fue completamente aleatorizado, con un esquema factorial $3 \times 2 \times 4$ (tres contenidos de humedad de la semilla: U1- U2U3; dos ambientes de almacenamiento: A1-A2 y cuatro períodos de almacenamiento: PA3-PA6-PA9-PA12) con cuatro repeticiones.

En el análisis de los datos se comprobó la normalidad y homogeneidad de la varianza mediante la prueba de Kolmogorov-Smirnov y la prueba de Cochran C, Hartley, Bartlett. Posteriormente, fueron sometidos a un análisis de varianza y las comparaciones entre las medias fue realizada mediante el Test de Tukey $(\mathrm{p}<0,05)$. Todos los análisis fueron realizados utilizando el paquete estadístico Statistica Versión 6.0 (Statsoft 2001).

\section{RESULTADOS Y DISCUSIÓN}

Los contenidos de humedad de las semillas no presentaron diferencias significativas entre sí, considerando la germinación para la determinación de la viabilidad inicial de las semillas (Cuadro 1).

Los resultados obtenidos muestran que independiente del contenido de humedad inicial, las semillas presentaron una germinación mayor del $87 \%$, resultados que coinciden con lo señalado por Santos et al. (1999) y Martins et al. (2005) quienes obtuvieron máxima germinación en semillas de papaya recién cosechadas. Sin embargo, Viggiano et al. (2000a) verificaron un bajo porcentaje de germinación en semillas de papaya recién cosechadas, lo que fue atribuido a la dormancia postcosecha. Lo anterior fue confirmado por Aroucha et al. $(2004,2007)$ en semillas del cv. Golden y el híbrido Tainung-1. Por otra parte, no coinciden con los obtenidos por Ellis et al. (1991). Dichos autores al secar las semillas de papaya a contenidos de humedad entre $5-11 \%$, verificaron una cierta dormancia, sugiriendo una sensibilidad a la desecación. Los resultados obtenidos sugieren que las pérdidas en la calidad fisiológica de las mismas dependerán principalmente de los efectos del contenido de humedad, el ambiente de conservación y el período de almacenamiento sobre el metabolismo celular de las semillas.

El análisis mostró efecto significativo $(\mathrm{p}<0,05)$ del contenido de humedad de la semilla, del ambiente de conservación, del período de almacenamiento y de la interacción entre estos factores (Cuadro 2).

Cuadro 1. Resultados medios de la germinación de las semillas de papaya cv Maradol Roja después de secadas y antes del almacenamiento. La Habana, Cuba. Noviembre 2006.

\begin{tabular}{cccc}
\hline $\begin{array}{c}\text { Contenido de humedad } \\
\text { inicial }(\boldsymbol{\%})\end{array}$ & $\begin{array}{c}\text { Porcentaje de emergen- } \\
\text { cia de las plántulas }(\%)\end{array}$ & $\begin{array}{c}\text { Altura promedio de la } \\
\text { planta }(\mathbf{c m})\end{array}$ & $\begin{array}{c}\text { Peso fresco de la } \\
\text { planta (g/planta) }\end{array}$ \\
\hline 9,26 & $85,5 \pm 7,634 \mathrm{NS}$ & $7,283 \pm 0,807 \mathrm{NS}$ & $4,908 \pm 0,437 \mathrm{NS}$ \\
10,56 & $87,5 \pm 6,761 \mathrm{NS}$ & $6,905 \pm 0,565 \mathrm{NS}$ & $4,343 \pm 0,590 \mathrm{NS}$ \\
12 & $88,0 \pm 3,829 \mathrm{NS}$ & $7,507 \pm 0,924 \mathrm{NS}$ & $4,112 \pm 0,246 \mathrm{NS}$ \\
$\mathrm{CV}$ & 2,084 & 10,89 & 12,29 \\
ES & 1,782 & 0,160 & 0,111 \\
\hline
\end{tabular}

NS: No significativo, CV: Coeficiente de variación, ES: Error estándar. 
Cuadro 2. Valores medios del porcentaje de germinación en los ensayos de semillas de papaya con diferentes contenidos de humedad, en dos ambientes de conservación durante cuatro períodos de almacenamiento. La Habana, Cuba. Período noviembre 2006-enero 2008.

\begin{tabular}{cccccc}
\hline \multirow{2}{*}{$\begin{array}{c}\text { Ambiente de } \\
\text { conservación }\end{array}$} & $\begin{array}{c}\text { Contenido de } \\
\text { humedad (\%) }\end{array}$ & Tres meses & Seis meses & Nueve meses & Doce meses \\
\cline { 3 - 6 } & 9,26 & $62,5 \pm 25,40 \mathrm{ab} *$ & $39,5 \pm 16,77 \mathrm{bc}$ & $76,5 \pm 8,00 \mathrm{ab}$ & $90,5 \pm 26,0 \mathrm{a}$ \\
& 10,56 & $57,5 \pm 5,03 \mathrm{ab}$ & $55,5 \pm 17,39 \mathrm{ab}$ & $81,5 \pm 11,01 \mathrm{ab}$ & $89,5 \pm 2,345 \mathrm{a}$ \\
& 12 & $62,5 \pm 14,04 \mathrm{ab}$ & $58,5 \pm 19,73 \mathrm{ab}$ & $69,5 \pm 6,83 \mathrm{ab}$ & $88,9 \pm 15,09 \mathrm{a}$ \\
\hline $4^{\circ} \mathrm{C}$ & 9,26 & $77,5 \pm 22,94 \mathrm{ab}$ & $69,5 \pm 21,75 \mathrm{ab}$ & $20,5 \pm 30,5 \mathrm{c}$ & $40,0 \pm 2,123 \mathrm{c}$ \\
& 10,56 & $62,5 \pm 25,81 \mathrm{ab}$ & $61,5 \pm 23,63 \mathrm{ab}$ & $27,5 \pm 6,83 \mathrm{c}$ & $17,5 \pm 23,40 \mathrm{c}$ \\
& 12 & $68,5 \pm 16,32 \mathrm{ab}$ & $49,5 \pm 27,39 \mathrm{ab}$ & $30,0 \pm 0,564 \mathrm{c}$ & $10,0 \pm 0,897 \mathrm{c}$ \\
\hline $\mathrm{CV}(\%)$ & 55,78 & & & & \\
$\mathrm{ES}$ & 3,13 & & & & \\
\hline
\end{tabular}

* Medias seguidas por letras iguales, no difieren entre sí por la Prueba de Tukey $(\mathrm{p}<0,05)$.

CV: coeficiente de variación, ES: Error estándar.

Se apreció una tendencia a la disminución de la germinación de las semillas de papaya del cv. Maradol Roja en los primeros seis meses del almacenamiento, alcanzándose los valores más bajos del porcentaje de germinación $(55,6 \%)$. A partir de los nueve meses se observó una mejoría significativa en la germinación, con valores de $67,7 \%$ y $77,5 \%$ a los nueve y doce meses, respectivamente.

Los resultados obtenidos durante los primeros seis meses de almacenamiento son similares a los señalado por Viggiano (1999) y Martins et al. (2004), donde las semillas de papaya después de almacenadas por cuatro meses presentaron reducción de la germinación y el vigor. Sin embargo, no coinciden con Tokuhisa et al. (2007) quienes verificaron mayor velocidad de germinación cuando las semillas permanecieron almacenadas por tres y seis meses.

Los valores observados a los nueves meses de almacenamiento no coinciden con lo señalado por Berbert et al. (2008), quienes plantean que los porcentajes de germinación para semillas con contenidos de humedad de $12,6 \%$ y $7,7 \%$, almacenadas después de ocho meses fueron similares, no encontrándose efecto significativo del secado de las semillas a diferentes contenidos de humedad.

Las semillas almacenadas a $15^{\circ} \mathrm{C}$, con contenidos de humedad de $10,56 \%$ y $12 \%$, presentaron mayor germinación en un periodo más largo de almacenamiento (aproximadamente doce meses) en relación a los demás períodos. Mientras que las semillas con esos mismos contenidos de humedad y mantenidas a $4^{\circ} \mathrm{C}$, exhibieron mejores porcentaje de germinación en un periodo menor, en torno a los tres meses de almacenamiento. Contrario a lo que afirman Carvalho y Nakagawa (2000), que para la gran mayoría de las especies, sus semillas serán mejor conservadas cuando menor sea la temperatura del aire de almacenamiento.

En el caso de las semillas con 9,26\% de humedad y almacenadas a $15^{\circ} \mathrm{C}$ se conservaron mejor, probablemente en función de su bajo grado de humedad. Estos resultados concuerdan con Viggiano et al. (2000b) según los cuales, semillas almacenadas en embalajes impermeables deben secarse a niveles más bajos de humedad. En estas condiciones, las semillas presentan baja actividad respiratoria y en consecuencia se deterioran más lentamente. Sin embargo, cuando las mismas fueron mantenidas a $4^{\circ} \mathrm{C}$, a los doce meses de almacenamiento presentaron germinación significativamente inferior a las semillas con $10,56 \%$ y $12 \%$ de humedad. Resultados similares fueron observados por Althoff y Carmona (1999) y Viggiano (1999), donde las temperaturas más bajas (menores de $10^{\circ} \mathrm{C}$ ) de almacenamiento no favorecen la germinación posterior de las semillas.

Según Berbert et al. (2008) el alto contenido de agua de las semillas es una de las principales causas 
de pérdida del poder germinativo y vigor de estas durante el almacenamiento, pues favorece procesos de deterioro. Es por ello que el secado de las semillas constituye una importante etapa en producción y su eficiencia podría reducir pérdidas existentes durante todo el proceso productivo, reflejando principalmente un buen establecimiento de la plantación.

Las semillas de papaya almacenadas en condiciones de $15^{\circ} \mathrm{C}$ y mantuvieron mayor porcentaje de germinación con respecto a las conservadas a bajas temperaturas $\left(4^{\circ} \mathrm{C}\right)$. Existieron diferencias en la germinación durante el período de almacenamiento. Resultados que coinciden con los obtenidos por Aroucha et al. $(2005,2007)$ para semillas del cv. Golden.

El almacenamiento de las semillas por un período de tiempo entre nueve y doce meses en un ambiente de conservación de $15^{\circ} \mathrm{C}$, propició una germinación superior mostrando que se requiere de este tiempo para superar una posible inducción de dormancia. Asimismo, estos resultados coinciden con los obtenidos por Aroucha et al. (2004) en semillas de papaya del cv. Golden y el híbrido Tainung 01, donde fue necesario un período de almacenamiento entre ocho a dieciseis meses, respectivamente para que las mismas obtuvieran más de un $80 \%$ de germinación.

Las semillas de papaya almacenadas en ambiente frío y en embalajes impermeables, los cuales impiden el intercambio de gases con el ambiente, presentan mayor resistencia a la superación natural de la dormancia (Viggiano et al. 2000). Por otra parte, de acuerdo a lo planteado por Ruggiero y Leonel (1988) la expresión del potencial de germinación y conservación de las semillas están, posiblemente, relacionado con los sistemas de control de entrada del agua en el interior, así como, la presencia de sustancias endógenas reguladoras del crecimiento existentes en las estructuras seminales.Ambos caracteres estudiados presentaron diferencias significativas para el efecto aislado de la condición ambiental de conservación y del periodo de almacenamiento. Se aprecia que la temperatura ambiental fue de fundamental importancia para la preservación de la calidad fisiológica de las semillas. La interacción condición temperatura $\mathrm{x}$ periodo de almacenamiento fue la más importante.

Por otra parte, hubo dependencia entre la humedad, temperatura ambiental y el período de almacenamiento para la masa de materia verde, así como las interacciones entre humedad $\mathrm{x}$ periodo de almacenamiento y humedad $\mathrm{x}$ temperatura ambiental también fueron significativas. De esta forma, la respuesta de los diferentes contenidos de humedad estudiados dependió del efecto de la condición ambiental de almacenamiento, básicamente de la humedad relativa del aire, debido a su relación directa con la semilla.

Estos resultados coinciden con lo señalado por Araujo et al. (1999) en un estudio realizado sobre la influencia de los factores: humedad y temperatura de almacenamiento en la conservación de semillas de papaya. A su vez, son similares a los obtenidos por Viggiano et al. (2000b) en la conservación de semillas de papaya en función del grado de humedad, tipo de embalaje y el ambiente de almacenamiento.

Las semillas almacenadas en condiciones de $15^{\circ} \mathrm{C}$ alcanzaron una mayor altura de la planta en un periodo de almacenamiento de nueve meses sin presentar diferencias con relación a los contenidos de humedad (Cuadro 3). Al contrario, de lo que ocurrió con las semillas almacenadas en condiciones de $4^{\circ} \mathrm{C}$, donde los valores alcanzados para periodos de almacenamiento más largo (entre nueve y doce meses) fueron mínimos.

Considerando la masa de materia verde, las semillas con contenidos de humedad 9,26\%, 10,56\% y $12 \%$ almacenadas en condiciones de $4^{\circ} \mathrm{C}$ en un periodo de tres meses mostraron los valores más altos para este carácter, a diferencia de las almacenadas en estas mismas condiciones pero por un periodo de doce meses, donde se obtuvieron los valores más bajos para la masa de materia verde de la planta (Cuadro 4).

De manera general, los resultados de las pruebas de germinación de las semillas confirmaron la presencia de dormancia en semillas de papaya durante el almacenamiento, requiriéndose de periodos más largos para superar una posible inducción de dicho proceso provocada por las condiciones de conservación. La superación parcial de la probable dormancia de estas semillas, después de dicho periodo, posiblemente, esté asociada a una mayor concentración de las sustancias inhibidoras de la germinación (Chow y Lin 1991).

La germinación de las semillas de papaya disminuyó en los primeros seis meses del almacenamiento, después de dicho periodo se evidencia un aumento significativo en la germinación independiente del contenido de humedad de la semilla.

Las semillas con contenidos de humedad entre $10-12 \%$ y almacenadas en condiciones de $15^{\circ} \mathrm{C}$, presentaron mayor germinación en periodos más largos de almacenamiento (aproximadamente doce meses). 
Cuadro 3. Valores medios de la altura de la planta en los ensayos de semillas de papaya almacenadas en dos temperaturas ambientales durante cuatro períodos de almacenamiento. La Habana, Cuba. Período noviembre 2006-enero 2008.

\begin{tabular}{ccccc}
\hline \multirow{2}{*}{$\begin{array}{c}\text { Ambiente de } \\
\text { conservación }\end{array}$} & Tres meses & Seis meses & Nueve meses & Doce meses \\
\cline { 2 - 5 } & $7,11 \pm 0,866 \mathrm{~b}^{*}$ & $4,64 \pm 4,779 \mathrm{bc}$ & $13,26 \pm 1,919 \mathrm{a}$ & $5,70 \pm 3,310 \mathrm{~b}$ \\
$15^{\circ} \mathrm{C}$ & $6,94 \pm 0,805 \mathrm{~b}$ & $7,96 \pm 8,551 \mathrm{~b}$ & $4,45 \pm 3,104 \mathrm{bc}$ & $0,00 \mathrm{c}$ \\
$4{ }^{\circ} \mathrm{C}$ & 8,18 & & & \\
$\mathrm{CV}(\%)$ & 0,518 & & & \\
$\mathrm{ES}$ & & & & \\
\hline
\end{tabular}

* Medias seguidas por letras iguales, no difieren entre sí por la Prueba de Tukey $(\mathrm{p}<0,05)$.

CV: Coeficiente de variación, ES: Error estándar.

Cuadro 4. Valores medios de la masa de materia verde de la planta en los ensayos de semillas de papaya con diferentes contenidos de humedad, en dos temperaturas ambientales, durante cuatro períodos de almacenamiento. La Habana, Cuba. Período noviembre 2006-enero 2008.

\begin{tabular}{cccccc}
\hline \multirow{2}{*}{$\begin{array}{c}\text { Ambiente de } \\
\text { conservación }\end{array}$} & $\begin{array}{c}\text { Contenido de } \\
\text { humedad }(\%)\end{array}$ & Tres meses & Seis meses & Nueve meses & Doce meses \\
\cline { 3 - 6 } & 9,26 & $5,26 \pm 0,235 \mathrm{a}$ * & $3,575 \pm 0,095 \mathrm{bc}$ & $3,15 \pm 0,655 \mathrm{~cd}$ & $1,50 \pm 0,081 \mathrm{e}$ \\
& 10,56 & $3,62 \pm 0,223 \mathrm{bc}$ & $3,225 \pm 0,095 \mathrm{~cd}$ & $3,75 \pm 0,310 \mathrm{bc}$ & $1,65 \pm 0,057 \mathrm{e}$ \\
& 12 & $3,65 \pm 0,514 \mathrm{bc}$ & $2,60 \pm 0,081 \mathrm{e}$ & $3,82 \pm 0,250 \mathrm{bc}$ & $1,90 \pm 0,081 \mathrm{e}$ \\
\hline $4^{\circ} \mathrm{C}$ & 9,26 & $5,44 \pm 0,058 \mathrm{a}$ & $3,95 \pm 0,129 \mathrm{~b}$ & $1,45 \pm 0,525 \mathrm{e}$ & $0,00 \mathrm{f}$ \\
& 10,56 & $5,47 \pm 0,061 \mathrm{a}$ & $4,12 \pm 0,095 \mathrm{~b}$ & $1,27 \pm 0,330 \mathrm{e}$ & $0,00 \mathrm{f}$ \\
& 12 & $5,51 \pm 0,370 \mathrm{a}$ & $5,02 \pm 0,095 \mathrm{a}$ & $0,00 \mathrm{f}$ & $0,00 \mathrm{f}$ \\
\hline $\mathrm{CV}(\%)$ & 55,78 & & & & \\
$\mathrm{ES}$ & 3,13 & & & & \\
\hline
\end{tabular}

* Medias seguidas por letras iguales, no difieren entre sí por la Prueba de Tukey $(\mathrm{p}<0,05)$.

CV: Coeficiente de variación, ES: Error estándar.

Sin embargo, cuando fueron mantenidas a $4^{\circ} \mathrm{C}$, exhiben mejores porcentaje de germinación en periodos menores de almacenamiento (aproximadamente tres meses).

La ocurrencia de dormancia en semillas de papaya durante el almacenamiento, puede ser superada por periodos de nueve y doce meses independientemente del contenido de humedad de esta pero teniendo en cuenta el ambiente de conservación.

\section{AGRADECIMIENTOS}

Los autores agradecen el apoyo y la experiencia brindada por el Dr. Roberto Ferreira Da Silva del Laboratorio de Fitotecnia, UENF, Brasil y por su contribución en la revisión del manuscrito. A la Empresa Productora y Comercializadora de Semillas por el financiamiento brindado al proyecto Conservación de semillas de papaya, de cual son parte estos resultados. 


\section{LITERATURA CITADA}

Althoff, MA; Carmona, R. 1999. Conservação de sementes de mamão (Carica papaya L. - Caricaceae). Revista Brasileira de Sementes 21(1):151-156.

Araujo, RC; Sampaio, LS; Costa, JA. 1999. Condición ambiental, contenido de agua y embalaje en la viabilidad y en el vigor de semillas de papaya. Revista Brasileira de Sementes 21(2):194-202.

Aroucha, EMM; Silva, RF; Vieira, RF; Viana, AP; Freitas, SP. 2004. Influência do estádio de maturação dos frutos e periodo de armazenamento das sementes no vigor das sementes de mamão dos grupos Solo e Formosa. In Reunião de Pesquisa do Frutimamão, Campos de Goytacazes, Anais. Campos de Goytacazes: UENF, 2004. Brasil. p. 71-75.

Aroucha, EMM; Silva, RF; Oliveira, JG; Viana, AP; Gonzaga, MP. 2005. Época de colheita e periodo de repouso dos frutos de mamão (Carica papaya L.) cv. Golden na qualidade fisiológica das sementes. Ciência Rural 35(3):537-543.

Aroucha, MM; Da Silva, FR; Balbinot, E; Nunes, SHG. 2007. Qualidade fisiológica de semente de mamão após o armazenamento dos frutos e de sementes. Revista Caatinga 20(3):136-143.

Bass, LN. 1975. Seed storage of Carica papaya L. HortScience 10(3):232-234.

Berbert, AP; Carlesso, OV; Da Silva, FR; Araújo, FE; Thiébaut, LTJ; De Oliveira, RTM. 2008. Qualidade fisiológica de semente de mamão em função da secagem e do armazenamento. Revista Brasileira de Sementes 30(1):23-25.

Carvalho, NM; Nakagawa, J. 2000. Sementes: ciencia, tecnología y producción. 4 ed. Campinas FUNEP, Brasil. $588 \mathrm{p}$.

Chow, YJ; Lin, CH. 1991. p-Hydroxybenzoic acid as the major phenolic germination inhibitor of papaya seed. Seed Science and Technology 19:167-174.

Ellis, RH; Hong, TD; Roberts, EH. 1991. Effect of storage temperature and moisture on the germination of papaya seeds. Seed Science Research 1:69-72.

Lopes, ML; Souza, MC. 2008. Efeitos da giberelina e da secagem no condicionamento osmótico sobre a viabilidade e o vigor de sementes de mamão (Carica papaya L.). Revista Brasileira de Sementes 30(1):181-189.
Martins, GN; Silva, RF; Araújo, EF; Vieira, HD; Viana, AP. 2004. Influência do tamanho do fruto, do peso específico e do periodo de armazenamento na qualidade fisiológica de sementes de mamão cv Golden. Revista Brasileira Armanz. 29(2):98-103.

Martins, GN; Silva, RF; Araújo, EF; Pereira, MG; Vieira, HD; Viana, AP. 2005. Influência do tipo de fruto, peso específico das sementes e periodo de armazenamento na qualidade fisiológica de sementes de mamão do grupo Formosa. Revista Brasileira de Sementes 27(2): 12-17.

MINAG (Ministerio de la Agricultura). 1996. Reglas para el análisis de las semillas. Manual de tecnología para la producción de semillas y frutas frescas para la exportación. Ed. MINAG, Cuba. 45 p.

Ramos, R; Ramos, JE. 2002. Instrucciones técnicas para el cultivo de la papaya Maradol Roja. Manual técnico Ed. Empresa de Semillas, MINAG. Cuba. 34 p.

Ruggiero, D; Leonel, S. 1988. Mamão. Jaboticabal: Departamento de Horticultura FCAV-UNESP. Brasil. 321 p.

Santos, RCA; Sampaio, LSV; Costa, JA. 1999. Condição ambiental, teor de água e embalagem na viabilidade e no vigor de sementes de mamão. Revista Brasileira de Sementes 21(2): 194-202.

Tokuhisa, D; Dias, SFCD; Alvarenga, ME; Dias, SAL; Marin, DLS. 2007. Tratamentos para superação da dormência em sementes de mamão. Revista Brasileira de Sementes 29(1):131-139.

Viggiano, JR. 1999. Influência do teor de umidade, tipo de embalagem e ambiente de armazenamento na conservação de sementes de mamão (Carica papaya L.). Campos dos Goytacazes. Dissertação (Mestrado em Produção Vegetal) - Universidade Estadual do Norte Fluminense, Campos dos Goytacazes, Brasil. 67 p.

Viggiano, JR; Silva, RF; Vieira, HD. 2000a Ocorrência de dormência em sementes de mamão (Carica papaya L.). Sementes Online 1(1):6-10.

Viggiano, JR; Vieira, HD; Silva, RF; Araújo, EF; Viana, AP. 2000b. Conservação de sementes de mamão (Carica papaya L.) em função do grau de umidade, tipo de embalagem e ambiente de armazenamento. Revista Brasileira de Sementes 22(2):279-287.

Yahiro, M. 1979. Effects of seed-pretreatments on the promotion of germination in papaya, Carica papaya L. Memorial Faculty Agriculture Kagoshima University, Kogoshima 15:49-54. 